\title{
A POCKET MODEL FOR THE SURFACE TENSION OF COMPRESSED NUCLEI
}

\author{
M. BRACK \\ Institute for Theoretical Physics, University of Regensburg, D-8400 Regensburg, W.-Germany \\ and \\ W. STOCKER \\ Sektion Physik der Universität München, D-8046 Garching, W.-Germany \\ Received 15 March 1982
}

\begin{abstract}
The surface structure of compressed semi-infinite isoscalar nuclear matter is studied starting from a simple energy density functional and adding constraints equivalent to some external pressure. For a wide class of compression modes we derive analytical expressions for the surface tension $\sigma$ as a function of the (de)compressed bulk density $n_{\mathrm{c}}$. The $\delta\left(n_{0}\right)=0$ theorem is verified for all modes. The model is tested with numerical calculations using realistic Skyrme-ETF energy density functionals including spin-orbit and effective mass contributions. They confirm the simple analytical model results almost quantitatively. The mode which leads to an antiscaled density (increasing surface diffuseness with increasing bulk density) leads to minimum values for $\sigma$ around $n_{0}$. The implications of the static model for the compression modes in finite nuclei are discussed.
\end{abstract}

\section{Introduction}

The problem of the structure of compressed nuclear matter occurs e.g. in the astrophysical question of the energy of nuclei immersed in an external gas of neutrons, electrons, etc. It is also of interest in heavy ion reactions where at least during a short period of time regions of compressed nuclear matter are generated. At present, the best available experimental and theoretical information on the structure of compressed nuclei comes from the analysis of the breathing mode. The literature about this mode is summarized in several extensive reports ${ }^{1-3}$ ).

Despite the numerous experimental and theoretical data on the breathing mode, there does not seem to exist a definite answer to the question of how the nuclear density varies during the breathing vibrations. Several modes of density vibrations have been discussed, of which the most common ones are defined either by a scaling of the density or by the multiplication of the density by a constant factor. The influence of oscillations of the surface diffuseness has also been studied ${ }^{4}$ ). Of course, particle number conservation must be taken care of in all these cases. A mode corresponding to constraining the rms radius is investigated in ref. ${ }^{2}$ ) in the context 
of the discussion of zero- and first-sound in nuclear matter. Most of these modes can be correlated to special sum rules ${ }^{2,5}$ ).

When the density vibrates adiabatically and in such a way that at any stage there is a clear separation between a bulk and a surface region of the breathing nucleus, a liquid drop (leptodermous) expansion of the compressibility modulus $K_{\mathrm{A}}$ of finite nuclei seems to converge rapidly:

$$
K_{\mathrm{A}}=K_{\infty}+K_{\mathrm{g}} A^{-\frac{1}{3}}+\ldots
$$

asymmetry and Coulomb corrections may also be added ${ }^{1,6}$ ). In eq. (1) $K_{\infty}$ is the compressibility of infinite isoscalar nuclear matter and the surface coefficient $K_{\mathrm{s}}$ may be written as

$$
K_{\mathbf{q}}=4 \pi r_{0}^{2}\left\{22 \sigma_{0}+9 \bar{\sigma}_{0}+54 \frac{\sigma_{0}}{K_{\infty}} \dddot{e}_{0}\right\}
$$

where $r_{0}$ equals $\left(\frac{4}{3} \pi n_{0}\right)^{-7}, \sigma_{0}=\sigma\left(n_{0}\right)$ is the surface tension at the saturation density $n_{0}, \bar{\sigma}_{0}$ is its reduced second derivative with respect to the density $n$ at saturation:

$$
\bar{\sigma}_{0}=\tilde{\sigma}\left(n_{0}\right)=\left.n_{0}^{2} \frac{\mathrm{d}^{2}}{\mathrm{~d} n^{2}} \sigma(n)\right|_{n_{0}},
$$

and $\dddot{e}_{0}$ is the correspondingly defined third derivative of the binding energy per nucleon $e_{\infty}(n)$ in isoscalar infinite nuclear matter. Evidently, expression (3) depends on the function $\sigma(n)$ which itself depends on the mode of the vibrating nuclear density ${ }^{\dagger}$.

In the case of the rms radius constraint, the coefficients in the expansion (1) would have to be changed ${ }^{2}$ ) since the system does not stay leptodermous. There are also arguments that the expansion (1) in this case might converge only slowly ${ }^{6,7}$ ).

The aim of the present paper is to calculate in a simple but selfconsistent model the surface tension $\sigma(n)$, in the plane geometry of semi-infinite isoscalar nuclear matter, for a wide class of density variations corresponding to a (de)compression of the bulk and arbitrarily coupled changes of the surface diffuseness. We use a simplified energy density functional, for which the surface tension in the free (i.e. uncompressed) case can be obtained analytically ${ }^{8}$ ), including a standard parametrization of the bulk energy density $\varepsilon_{x_{0}}(n)$ and a Weizsācker type gradient term. We discuss the external constraints necessary to produce density compressions in a variational way and calculate the function $\sigma(n)$ which can again be found analytically for the whole class of density variations mentioned above. The results make transparent the

\footnotetext{
+ Eq. (2) was originally derived for the scaling model ${ }^{1}$ ); it holds, however, also for the whole class of compression modes discussed below [see especially the discussion after fig. 3].
} 
dependence of the quantity $\ddot{\sigma}_{0}$ eq. (3) on the various ways by which the surface might be coupled to the variations of the bulk density. With the help of numerical calculations using realistic Skyrme forces, we shall finally test the relevance of the results of our simple model.

\section{The free semi-infinite case}

Let us now describe a simple selfconsistent model for calculating the surface tension $\sigma$ of semi-infinite nuclear matter, which is defined as usual by

$$
\sigma=\int_{-\infty}^{+\infty} \mathrm{d} z\left\{\varepsilon[n]-a_{v} n(z)\right\}
$$

where we have chosen the density $n$ to vary along the $z$-axis. The simplest way to parametrize the total energy density $\varepsilon[n]$ is to write it as the sum of a bulk part $\varepsilon_{\infty}(n)$ and a gradient term:

$$
\begin{gathered}
\varepsilon[n]=\varepsilon_{\infty}(n)+\eta \frac{(\nabla n)^{2}}{n}, \\
\varepsilon_{\infty}(n)=n e_{\infty}(n)=n\left\{a_{v}+\frac{1}{18} K_{\infty}\left(\frac{n}{n_{0}}-1\right)^{2}\right\} .
\end{gathered}
$$

The bulk energy density $\varepsilon_{\infty_{0}}(n)$ eq. (6) comprises the three main properties of infinite nuclear matter more or less known from experiment: the binding energy per particle $a_{v}=e_{\infty}\left(n_{0}\right)$, the saturation density $n_{0}$ and the incompressibility $K_{\infty}$. The surface term in eq. (5) has been chosen to lead to a realistic exponential fall-off ${ }^{9}$ ) of the density $n(z)$ far from the surface, say for $z \rightarrow \infty$. The profile which minimizes the surface tension $\sigma$ eq. (4) is obtained through the variational principle $\delta \sigma / \delta n(z)=0$ which leads to the following Euler equation:

$$
-\eta\left[\left(\frac{y^{\prime}}{y}\right)^{2}-2 \frac{y^{\prime \prime}}{y}\right]=\frac{\partial \varepsilon_{\infty}(n)}{\partial n}-a_{v}
$$

where we have put $n(z)=n_{0} y(z)$ and primes denote derivation with respect to $z$. Eq. (7) with the form eq. (6) of $\varepsilon_{\infty}(n)$ can be solved analytically ${ }^{8}$ ) which is most easily done by substituting $y^{\prime}(z)=p(y)$ leading to a linear first-order differential equation in $p(y)$. The result for $y^{\prime}(z)$ is

$$
y^{\prime}(z)=\frac{1}{z^{\prime}(y)}=-\sqrt{y \frac{1}{\eta n_{0}}\left[\varepsilon_{\infty}(n)-a_{v} n\right]}=-\sqrt{\frac{K_{\infty}}{18 \eta}} y(1-y) .
$$


The sign in eq. (8) is chosen according to the boundary conditions $y(-\infty)=1$, $y(+\infty)=0$. Integrating the inverse of eq. (8) and inverting $z(y)$, one finds

$$
n(z)=\frac{n_{0}}{1+\mathrm{e}^{z / a_{0}}}
$$

with

$$
\alpha_{0}=\sqrt{\frac{18}{K_{\infty}} \eta}
$$

Inserting the density $n(z)$ eq. (9) into eq. (4), we obtain for the surface tension at saturation:

$$
\sigma_{0}=\frac{K_{\infty}}{18} n_{0} \frac{\alpha_{0}}{2}+\frac{1}{2 \alpha_{0}} \eta n_{0}=n_{0} \sqrt{\frac{K_{\infty} \eta}{18}}=\frac{K_{\infty}}{18} n_{0} \alpha_{0} .
$$

An expression equivalent to the right-hand side of eq. (11) has been derived recently from very general assumptions by one of us ${ }^{10}$ ). It may be worth noting that the bulk and surface terms of eq. (5) each contribute exactly one-half of the surface tension eq. (11). This would remain true if $\varepsilon_{\infty}(n)$ were any function of $n$ and the gradient term replaced by $g(n) \cdot \Delta n+h(n) \cdot(\nabla n)^{2}$ with arbitrary functions $g$ and $h$.

\section{The compressed case}

So far, we have obtained an expression for the surface tension at saturation, i.e. for the free semi-infinite system. As discussed in the introduction, we want to investigate how $\sigma$ varies when the nuclear matter in the bulk region is compressed or decompressed. We therefore must extend our variational calculation to obtain solutions with the boundary conditions

$$
n(-\infty)=n_{\mathrm{e}}, \quad n(+\infty)=0,
$$

where $n_{\mathrm{c}}$ may be different from $n_{0}$. We first point out that such solutions cannot be obtained without applying an external constraint on the density. In fact, replacing $a_{\mathrm{v}}$ in eq. (4) by $\varepsilon_{x_{0}}\left(n_{\mathrm{c}}\right) / n_{\mathrm{c}}$ and performing the variation $\delta \sigma / \delta n(z)=0$ leads to the differential equation

$$
y^{\prime}(z)=-\frac{1}{\alpha_{0}} y \sqrt{q^{2} y^{2}-2 q y+2 q-q^{2}},
$$


where we have put $n(z)=n_{\mathrm{c}} y(z)$ and

$$
q=\frac{n_{\mathrm{c}}}{n_{0}}
$$

No reasonable solution of eq. (13) with the boundaries eq. (12) can be found for $q \neq 1$. (A continuous solution $n(z)$ can be pieced together in the case of decompression, i.e. $n_{\mathrm{c}}<n_{0}$ only; it leads, however, to a logarithmic divergence of $\sigma\left(n_{0}\right)$ even though $\dot{\sigma}\left(n_{0}\right)$ is zero.)

We are therefore forced to include an external constraint in the variational equation. This may be done ${ }^{11}$ ) by adding an extra term to the energy density, i.e. replacing $\varepsilon[n]$ by

$$
\varepsilon_{\mathrm{c}}[n]=\varepsilon[n]+C[n]
$$

The term $C[n]$ can be understood to cause an external pressure which leads to a (de)compression of the bulk density from $n_{0}$ to $n_{\mathrm{c}}$ accompanied by some change of the surface structure. The constrained energy density $\varepsilon_{c}[n]$ eq. (15) must be subject to the generalized saturation condition

$$
\frac{\partial}{\partial n_{\mathrm{c}}}\left(\frac{\varepsilon_{\mathrm{c}}\left[n_{\mathrm{c}}\right]}{n_{\mathrm{c}}}\right)=\mathbf{0}
$$

which ensures the balance of internal and external pressure in the bulk region.

In constructing the constraint $C[n]$ we have been guided by the following considerations: (i) The constraint should be constant in the bulk region and fall off in the surface region, thus leaving the surface as free as possible. This can be achieved by taking $C[n]$ to be some polynomial in $n$. (ii) The class of density variations caused by the constraint should be flexible enough to include various couplings of bulk density and surface variations, in particular also the scaling mode and that of changing $n_{\mathrm{c}}$ with fixed surface diffuseness. (iii) The variational equation for $\varepsilon_{c}[n]$ eq. (15), which will take the form

$$
\frac{\delta}{\delta n(z)} \int_{-\infty}^{+\infty}\left\{\varepsilon_{\mathrm{c}}[n]-\frac{\varepsilon_{\mathrm{c}}\left[n_{\mathrm{c}}\right]}{n_{\mathrm{c}}} n(z)\right\} \mathrm{d} z=0,
$$

should be analytically soluble and lead to simple expressions for $\sigma(n)$.

These conditions can be met by the following form of $C[n]$ (with $\beta$ being any real number):

$$
C[n]=\frac{K_{\infty}}{18} \frac{n^{2}}{n_{\mathrm{c}}}\left[\frac{n}{n_{\mathrm{c}}}\left(q^{-2 \beta}-q^{2}\right)-2 q^{-2 \beta}+2 q\right],
$$


where $q$ is given by eq. (14). With this choice, eq. (17) leads, indeed, after one integration using the same substitution as in the free case, to

$$
y^{\prime}(z)=-\frac{1}{\alpha_{0} q^{\beta}} y(1-y)
$$

We thus obtain again Fermi-type density profiles

$$
n(z)=\frac{n_{\mathrm{c}}}{1+\mathrm{e}^{2 / \alpha_{\mathrm{c}}}}
$$

with

$$
\alpha_{c}=\alpha_{0} q^{\beta}=\alpha_{0}\left(\frac{n_{\mathrm{c}}}{n_{0}}\right)^{\beta}
$$

The surface tension is still given by eq. (4) replacing $a_{\mathrm{v}}$ by $\varepsilon\left[n_{\mathrm{c}}\right] / n_{\mathrm{c}}-$ thus leaving out the constraint $C[n]-$ and is easily found to be

$$
\sigma\left(n_{\mathrm{c}}\right)=\sigma(q)=\sigma_{0} \frac{1}{2}\left[4 q^{\beta+2}-3 q^{\beta+3}+q^{1-\beta}\right] .
$$

Note that the familiar $\dot{\sigma}_{0}=\left.\sigma^{\prime}(q)\right|_{q=1}=0$ theorem ${ }^{12}$ ) is fulfilled for any value of $\beta$ [see also ref. $\left.{ }^{11}\right)$ ]. The second derivative of $\sigma(q)$ at saturation is then

$$
\tilde{\sigma}_{0}=\left.\frac{\mathrm{d}^{2} \sigma}{\mathrm{d} q^{2}}\right|_{q=1}=\sigma_{0}\left(\beta^{2}-2 \beta-5\right) .
$$

The parameter $\beta$ triggers the degree to which the variation of the bulk density is coupled to a variation of the surface diffuseness $\alpha_{\mathrm{c}}$ as given by eq. (21). The familiar scaling mode ${ }^{1,2,4.5}$ ) is obtained with $\beta=-\frac{1}{3}$. Positive values of $\beta$ lead to some kind of antiscaling of various degree, whereas $\beta=0$ means multiplication of the density by a constant factor $q$. We see from eq. (23) that $\bar{\sigma}_{0}$ depends quadratically on $\beta$; thus also the surface compressibility $K_{\mathrm{s}}$ eq. (2) will depend on the chosen mode.

We now address the question which mode might be energetically most favourable. From purely static considerations we are led to choose the mode for which $\vec{\sigma}_{0}$ is as negative as possible; this leads to the largest gain of (surface) energy, on the average, under the oscillations of $n_{\mathrm{c}}$ around $n_{0}$. Within our class of modes, we find that $\beta=1$ minimizes the quantity $\bar{\sigma}_{0}$ eq. (23), giving it the value

$$
\bar{\sigma}_{0}=-6 \sigma_{0}=-\frac{1}{3} K_{\infty} n_{0} \alpha_{0} \quad(\beta=1) .
$$

This is also illustrated in fig. 1 where we show the functions $\sigma(q)$ eq. (22) around the saturation point $(q=1)$ for various values of $\beta$. The curve with $\beta=1$ is lowest, at least for values of $q$ not too different from unity. 


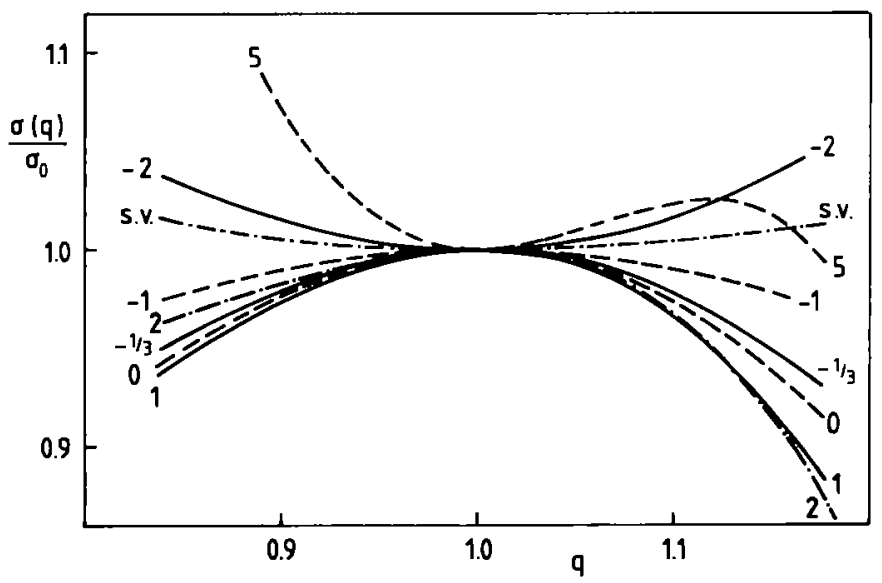

Fig. 1. Plots of the relative surface tension versus the relative bulk density $q$, according to eq. (22), for various modes. The numbers indicate the values of $\beta$ as defined through eqs. (20), (21). The curve labelled "s.v." corresponds to a pure surface vibration, see eq. (26).

The result in eq. (24) is in perfect agreement with a similar formula derived recently ${ }^{13}$ ) from general considerations assuming a free surface. Although the constraint $C[n]$ eq. (18) is not entirely inactive in the surface region, it can be seen qualitatively to be least important there for $\beta \simeq 1$ from fig. 2 , where we have

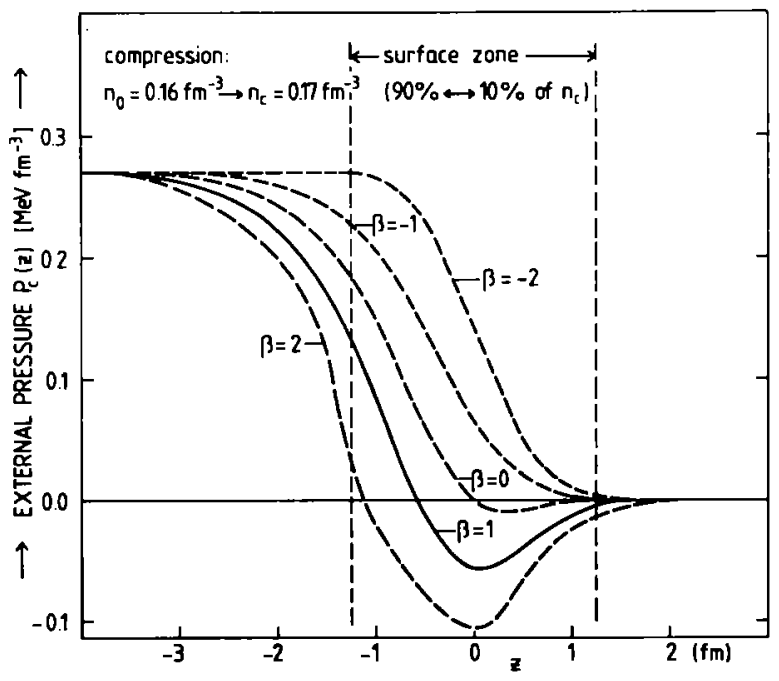

Fig. 2. Plots of the external pressure $P_{c}(z)$, eq. (25), for various values of $\beta$, corresponding to a compression of the bulk density from $n_{0}=0.16 \mathrm{fm}^{-3}$ to $n_{\mathrm{c}}=0.17 \mathrm{fm}^{-3}$ (i.e. $q=1.0625$ ). A compressibility of $K_{\infty}=216 \mathrm{MeV}$ has been used. The surface region, in which the density falls from $90 \%$ to $10 \%$ of $n_{c}$, is indicated by vertical dashed lines. 
plotted the external pressure $P_{c}(z)$, defined locally by

$$
P_{\mathrm{c}}(z)=-n^{2} \frac{\mathrm{d}}{\mathrm{d} n}\left[\frac{C[n]}{n}\right]=\frac{K_{\infty}}{9 n_{\mathrm{c}}} n^{2}\left[\frac{n}{n_{\mathrm{c}}}\left(q^{2}-q^{-2 \beta}\right)+q^{-2 \beta}-q\right],
$$

for different values of $\beta$. Indeed, the absolute value of $P_{c}$ averaged over the surface region is seen to be smallest for $\beta \simeq 1$.

A mode which is not contained in the family defined above is that of a pure surface vibration with constant bulk density $n_{0}$ [see e.g. ref. $\left.{ }^{4}\right)$ ]. It must, of course, be obtained through a constraint which is only effective in the surface region. In fact, choosing $C[n]=\eta^{\prime}(\nabla n)^{2} / n$ and suitably adjusting $\eta^{\prime}$, we are led to density profiles of the form of eq. (20) with $n_{\mathrm{c}}=n_{0}$ and $\alpha_{\mathrm{c}}=\alpha_{0} q$. In this case, the surface tension $\sigma(q)$ is found to be

$$
\sigma(q)=\sigma_{0} \frac{1}{2}\left[q+q^{-1}\right] ;
$$

the corresponding curve is shown in fig. 1 with the label "s.v." (surface vibration). This surface mode was analysed for finite nuclei by Jensen and Larsen ${ }^{4}$ ). In a dynamical model using realistic Skyrme forces, they studied mixtures of scaling and pure surface vibrations. They found that the lowest normal isoscalar mode in the two-dimensional space spanned by these two types of vibrations coincides, at least for large nuclei, with the scaling mode.

Summarizing up to this point, we have formulated a simple analytical variational model for obtaining the dependence of the surface tension on the (de)compressed bulk density, including a wide class of modes by which surface vibrations are coupled to the changes of the bulk density. The energetically most favourable of all these modes is expected to be the one with $\beta=1$ which we may call an antiscaling mode, since for this mode the surface diffuseness increases with increasing bulk density, in contrast to the standard scaling mode $\left(\beta=-\frac{1}{3}\right)$. Note, however, that the differences between the curves in fig. 1 for $\beta=-\frac{1}{3}, 0$ or 1 are rather small for small amplitude density vibrations $(q \approx 1)$. Therefore, dynamical effects such as caused e.g. by variable inertial masses corresponding to the different modes, might alter somewhat the conclusions.

\section{Comparison to numerical calculations with realistic energy density functionals}

In order to test the results of our simple pocket model, we shall in the following compare them to numerical results obtained with realistic energy densities. We employ the so-called extended Thomas-Fermi (ETF) method which recently has been successfully used in connection with Skyrme-type effective interactions to calculate average nuclear properties; its results have been shown to be in quantitative agreement 
with microscopically averaged Hartree-Fock calculations $\left.{ }^{14-16}\right)$. Hereby, corrections to the kinetic and spin-orbit energies up to fourth order in the ETF density gradient expansion must be included; they are particularly important for obtaining the correct average deformation properties (e.g. fission barriers) of finite nuclei. As shown in ref. ${ }^{15}$ ) for various Skyrme forces, the surface energy eq. (4) is well minimized (within less than $1 \%$ ) by density profiles of the form

$$
n(z)=\frac{n_{0}}{\left(1+\mathrm{e}^{z / \alpha_{0}}\right)^{y}}
$$

with typical values $\alpha_{0} \simeq 0.5-0.65 \mathrm{fm}$ and $\gamma \simeq 1.3-1.8$.

For our present investigations, we chose three energy densities $\varepsilon[n]$ with realistic compressibilities and surface tensions: those of the Skyrme forces Ska ${ }^{17}$ ) and SkM $^{*}$ $\left[\right.$ refs. $\left.{ }^{15,16}\right)$ ], and the energy density of Tondeur ${ }^{18}$ ). [The Skyrme force SkM* is a modified version of the force SkM which was recently adjusted to reproduce the giant monopole, dipole and quadrupole resonances in RPA type calculations ${ }^{19}$ ); the parameter set $\mathrm{SkM}^{*}$ has the same nuclear matter properties as SkM but leads to much improved Hartree-Fock binding energies and radii of stable nuclei and to the correct average fission barriers of actinide nuclei ${ }^{15,16}$ ).] We give the values of $K_{\infty}, n_{0}$ and $\sigma_{0}$ of these forces in table 1 .

Using the optimized values of $\alpha_{0}$ and $\gamma$ (also shown in table 1), we varied the density profiles eq. (27) according to the modes discussed above, replacing $n_{0}$ and $\alpha_{0}$ by $n_{0} q$ and $\alpha_{0} q^{\beta}$, respectively, and computed numerically the quantity $\ddot{\sigma}_{0}=\mathrm{d}^{2} \sigma / \mathrm{d} q^{2} l_{q}=1$. The results are shown as functions of $\beta$ in fig. 3 by the dashed-dotted curves; the solid line represents the prediction eq. (23) of the pocket model. The close agreement is very astonishing: all three curves have their maximum close to $\beta=1$ and coincidence to within $\sim 10 \%$ with the pocket model curve in the interesting range $-\frac{1}{3} \leqslant \beta \leqslant 2$. This demonstrates that our pocket model contains the necessary ingredients for the quantity $\ddot{\sigma}(\beta) / \sigma_{0}$ which

TABLE 1

Saturation and surface properties of the Skyrme forces SkM*15.16), Ska ${ }^{17}$ ) and the Tondeur energy density ${ }^{18}$ )

\begin{tabular}{|c|c|c|c|c|c|c|c|c|c|}
\hline \multirow{2}{*}{ Force } & \multirow{2}{*}{$\begin{array}{c}K_{x} \\
(\mathrm{MeV})\end{array}$} & \multirow{2}{*}{$\begin{array}{c}n_{0} \\
\left(\mathrm{fm}^{-3}\right)\end{array}$} & \multirow{2}{*}{$\begin{array}{c}\alpha_{0} \\
(\mathrm{fm})\end{array}$} & \multirow{2}{*}{$\gamma$} & \multirow{2}{*}{$\begin{array}{c}\sigma_{0} \\
\left(\mathrm{MeV} \cdot \mathrm{fm}^{-2}\right)\end{array}$} & \multirow{2}{*}{$\begin{array}{c}K \\
(\beta=1) \\
(\mathrm{MeV})\end{array}$} & \multicolumn{3}{|c|}{$-K_{y} / K_{x}$} \\
\hline & & & & & & & $\beta=-\frac{1}{3}$ & $\beta=0$ & $\beta=1$ \\
\hline Ska & 263.2 & 0.1554 & 0.6019 & 1.700 & 1.107 & -733 & 1.08 & 1.80 & 2.79 \\
\hline Ton & 235.9 & 0.1590 & 0.6221 & 1.775 & 1.099 & -689 & 1.09 & 1.86 & 2.92 \\
\hline SkM $^{*}$ & 216.7 & 0.1603 & 0.6337 & 1.782 & 1.051 & -631 & 1.07 & 1.86 & 2.91 \\
\hline
\end{tabular}

The surface parameters for the semi-infinite case $\alpha_{0}, \gamma$ and $\sigma_{0}$ - see eqs. (4) and (27) - are obtained in numerical ETF calculations as described in ref. ${ }^{15}$ ). See text for the calculation of the surface compressibilities $K$, as functions of $\beta$. 


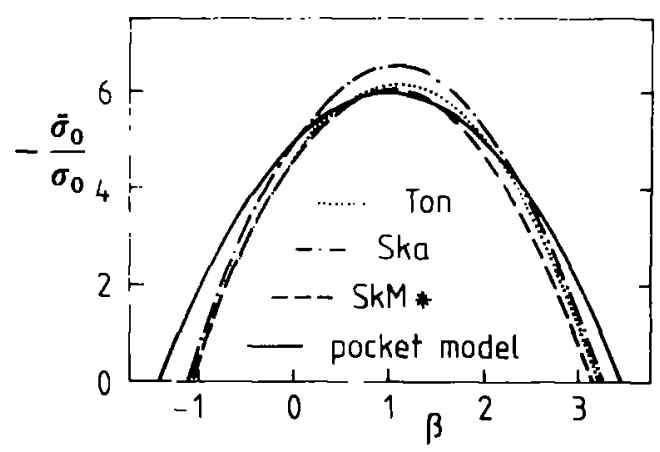

Fig. 3. Second reduced derivative of the surface tension with respect to the density at the saturation point, eq. (3), plotted versus the parameter $\beta$ of the compression mode. The solid curve shows the pocket model prediction eq. (23). The other curves are obtained numerically using three realistic energy densities (see table 1) containing spin-orbit and effective mass (for Ska, SkM+) contributions as well as second- and fourth-order ETF gradient corrections ${ }^{15}$ ).

apparently is nearly model independent. The close agreement is the more surprising that spin-orbit and variable effective mass contributions as well as higher order gradient corrections are all missing in the pocket model; although they modify crucially the surface tension $\sigma_{0}$, they appear to have little influence on $\dot{\sigma}(\beta) / \sigma_{0}$.

We can conclude from these results that the compression mode with $\beta=1$ is (adiabatically) favourable also when very realistic forces are used. It is therefore of interest to discuss the consequence which this will have on the value of the surface compressibility $K_{\mathrm{s}}$ eq. (2) and, therefore, the total compressibility $K_{\mathrm{A}}$ of finite nuclei. We should note at this point that eq. (2) holds for all modes described by $\beta$, if $\bar{\sigma}_{0}$ is defined as in eq. (23). In fact, the derivations of eq. (2) given in refs. ${ }^{13,20}$ ) hold for any value of $\beta$. The only assumption is that the density is leptodermous, i.e. flat in the interiour part of the nucleus so that the total energy can be split up into a volume and a surface part. The expansion eq. (1) of $K_{\mathrm{A}}$ has been checked numerically within the ETF-Skyrme formalism ${ }^{20}$ ) for $\beta=0$. The convergence of eq. (1) was found to be very fast for nuclei with $N=Z$ and without Coulomb interaction; the curvature term $\sim A^{-2 / 3}$ was found to be practically negligible and the expression eq. (2) for $K_{\mathrm{a}}$ reproduced the slopes of the numerical results of $K_{\mathrm{A}}=9 n_{0}^{2}\left(\mathrm{~d}^{2} / \mathrm{d} n_{0}^{2}\right)(E / A)$, plotted versus $A^{-t}$, within a few percent. [See also a similar test in ref. $\left.{ }^{6}\right)$.]

Relying on this fast convergence, we have used eq. (2) to compute $K$, for the three forces given in table 1 as functions of $\beta$. We give in the same table the values of $K_{\mathrm{s}}$ obtained for $\beta=1$ and the ratios $-K_{\mathrm{s}} / K_{\mathrm{o}}$ for the three most interesting modes $\beta=-\frac{1}{3}$ (scaling), $\beta=0$ ("factor") and $\beta=1$ (antiscaling). The variation of $K_{z}$ for these cases is surprisingly large - by almost a factor of 3 - due to a strong cancellation between the first two terms on the right-hand side of eq. (2). However, in spite of this strong variation, the ratios $K_{\mathbf{g}} / K_{\infty}$ are practically identical for the 
three forces for each value of $\beta^{*}$. This can be qualitatively understood in our pocket model, combining eqs. (2), (11) and (23), and noticing that the correction proportional to $\dddot{e}_{0}$ in eq. (2) which changes $K_{\mathrm{s}}$ by $\sim 10-20 \%$, is roughly the same for all forces considered here. The values of $K_{\mathrm{s}} / K_{\mathrm{o}}$ given in table 1 can indeed be fitted within $\sim 3 \%$ by the formula

$$
K_{8} / K_{\infty}=-1.02\left(1.81+2 \beta-\beta^{2}\right) .
$$

The surface compressibility $K_{\mathrm{s}}$ is thus a very sensitive function of the compression mode. It might therefore be tempting to try to extract its value from experiments in order to learn about the nature of the breathing mode, i.e. about the value of $\beta$ in our parametrization. Unfortunately, extraction of the quantity $K_{\mathrm{A}}$ from the experimental giant monopole resonance (GMR) energies requires already the knowledge of the breathing mode itself. For scaling one obtains the familiar formula ${ }^{1,2}$ )

$$
E_{\mathrm{GMR}}=\sqrt{\frac{\hbar^{2}}{m} \frac{K_{\mathrm{A}}}{\left\langle r^{2}\right\rangle}}
$$

which is most frequently used to determine $K_{\mathrm{A}}$. Treiner et al. ${ }^{6}$ ) have analysed the most recent experimental data of the breathing mode. Although they show convincingly that the scaling mode, using $K_{\mathrm{s}}=-K_{x}$, allows for a consistent description of the data with a surprising accuracy, their fit of the data to the liquid drop expansion eq. (1) of $K_{\mathrm{A}}$ [see table 10 of ref. ${ }^{6}$ )] seems to indicate that values of $-K_{s} \gtrless 2 K_{\infty}$ are by no means excluded. It will therefore be decisive for further discussions of leptodermous compression modes other than the scaling mode, to know how the variation of the inertial parameters with $\beta$ will modify eq. (29). Studies in this direction are under way.

\section{Summary and conclusions}

Starting from a simple nuclear energy density functional we have investigated the structure of a (de)compressed plane system of nuclear matter by introducing constraints equivalent to an external pressure. Choosing a rather general class of constraints, we were able to treat analytically different modes for the coupling of the (de)compression of the bulk part to that of the surface region.

We could corroborate recent constrained Hartree-Fock calculations with realistic nuclear interactions ${ }^{11}$ ). Also, simple pocket formulae in refs. ${ }^{10,13}$ ), which contain only well-known ground-state properties, are confirmed in our approach. In

\footnotetext{
* The result $K_{1} \approx-K_{\infty}$ for the scaling mode is in agreement with the findings of ref. ${ }^{6}$ ).
} 
particular, we obtain a vanishing first derivative $\dot{\sigma}\left(n_{0}\right)$ for all (de)compression modes we have considered. Thus, the $\dot{\sigma}=0$ theorem originally proposed by Myers and Swiatecki ${ }^{12}$ ) only for the case of a compressed bulk, but a free surface, is shown to hold very generally in a variational treatment. Remarkably, $\sigma$ has generally a maximum at the saturation density; for rather exotic constraints which are extremely effective in the surface zone, however, $\sigma_{0}$ may also be a minimum.

In a static adiabatic description of the breathing mode, the density in the surface region rearranges itself at any stage of the vibration in such a way that the corresponding static surface tension is a minimum. It follows then that an antiscaled density is energetically favourable, i.e. a density whose surface diffuseness increases with increasing (compressed) bulk density. The energy gain in this mode $(\beta=1)$ compared to e.g. the scaling mode is, however, relatively small, so that dynamical effects (e.g. a strong dependence of the inertial mass parameters on $\beta$ ) may influence the nature of the vibration. Extending this treatment to the description of breathing vibrations of finite nuclei, care should be taken of the particle number conservation. Also, besides dynamical effects, other constraints which do not lead to a clear separation of the bulk from the surface region, e.g. the rms constraint mode ${ }^{2,6}$ ), should be taken into consideration.

Using two realistic Skyrme and the Tondeur energy density functionals including semiclassical ETF gradient corrections, we have numerically verified the simple model prediction for the dependence of $\bar{\sigma}_{0}$ on the vibration mode [see eq. (23)] and shown that this analytical formula is nearly model independent. Calculating the surface coefficient $K_{\mathrm{a}}$ in the liquid drop expansion of the finite-nucleus incompressibility $K_{\mathrm{A}}$ [see eq. (1)], we find a strong dependence of $K_{\mathrm{g}}$ on the nature of the breathing mode; this dependence is, again, practically model independent if $K_{\mathrm{s}}$ is measured in units of the infinite nuclear compressibility $K_{\infty}$ [see eq. (28)].

Extracting $K_{\mathrm{s}}$, using a dynamical treatment, from the experimentally measured giant monopole resonance energies could in principle give insight into the nature of the surface behaviour under the breathing oscillations. However, as often happens when considering energies alone, the conclusion might not be unique. Therefore, a careful analysis of the transition densities might give more insight about the various modes. Such an analysis was recently performed, but does not yet seem to yield unique conclusions about the degree to which surface oscillations are coupled to the bulk density oscillations ${ }^{21}$ ).

We are grateful to Prof. M. Pearson for his warm hospitality and for many stimulating discussions during our visit at the Laboratoire de Physique Nucléaire de l'Université de Montréal, where this investigation was started. We also acknowledge stimulating discussions with Drs. J. Treiner and H. P. Morsch. We are indebted to Dr. H.-B. Halkansson for letting us use his semi-infinite nuclear matter ETF code prior to publication. Grants from the Ministère des affaires intergouvernementales du Gouvernement du Québec and a travel grant of the Deutsche Forschungsgemeinschaft are gratefully acknowledged. 
Note added in proof: Preliminary numerical calculations within a hydrodynamical model equivalent to that of ref. ${ }^{4}$ ) seem to indicate that the inertial parameters corresponding to vibrations according to the above modes (with fixed $\beta$ ) increase with $\beta$ decreasing from $\beta=1$ to $\beta=-\frac{1}{3}$, such that the frequency of small oscillations is minimized for $\beta$ close to zero. Realistic calculations for finite nuclei are under way.

\section{References}

1) J. P. Blaizot, Phys. Reports 64 (1980) 171

2) B. K. Jennings and A. D. Jackson, Phys. Reports 66 (1980) 141

3) J. Speth and A. v.d. Woude, Reports on Progr. in Phys. 44 (1981) 719

4) A. S. Jensen and S. M. Larsen, Phys. Scripta 24 (1981) 534

5) O. Bohigas, A. M. Lane and J. Martorell, Phys. Reports 51 (1979) 267

6) J. Treiner, H. Krivine, O. Bohigas and J. Martorell, Nucl. Phys. A371 (1981) 253

7) J. M. Pearson, M. Farine and J. Coté, Phys. Rev. C26 (1982) 267

8) M. Brack, Habilitationsschrift, Grenoble 1977 (ILL-77 BR 346S)

9) R. A. Berg and L. Wilets, Proc. Phys. Soc. A68 (1955) 229

10) W. Stocker, Phys. Lett. 104B (1981) 339

11) M. Farine, J. Coté, J. M. Pearson and W. Stocker, Z. Phys., to appear; see also M. Farine, These de Doctorat, Université de Montreal (1981)

12) W. D. Myers and W. J. Swiatecki, Ann. of Phys. 55 (1969) 395

13) W. Stocker, Nucl. Phys. A342 (1980) 293

14) C. Guet, H.-B. Håkansson and M. Brack, Phys. Lett. $97 B$ (1980) 7

15) C. Guet, H.-B. Håkansson and M. Brack, preprint Regensburg, 1982, Nucl. Phys. A, to be submitted

16) J. Bartel, P. Quentin, M. Brack, C. Guet and H.-B. Hakansson, Nucl. Phys. A386 (1982) 79

17) S. Köhler, Nucl. Phys. A258 (1976) 301

18) F. Tondeur, Nucl. Phys. A315 (1978) 353

19) H. Krivine, J. Treiner and O. Bohigas, Nucl. Phys. A366 (1980) 155

20) M. Brack et al., 4th. Conf. on nuclei far from stability, Helsing $\phi r$ 1981 (CERN 81-09, Geneva) p. 65

21) H. P. Morsch et al., Phys. Rev. C22 (1980) 489;

H. P. Morsch, private communications 